\title{
New Neurons in the Adult Mammalian Brain: Synaptogenesis and Functional Integration
}

\author{
Hongjun Song, ${ }^{1}$ Gerd Kempermann, ${ }^{2}$ Linda Overstreet Wadiche, ${ }^{3}$ Chunmei Zhao, ${ }^{4}$ Alejandro F. Schinder, ${ }^{5}$ and \\ Josef Bischofberger ${ }^{6}$ \\ ${ }^{1}$ Institute for Cell Engineering, Departments of Neurology and Neuroscience, Johns Hopkins University School of Medicine, Baltimore, Maryland 21205, \\ ${ }^{2}$ Max Delbrück Center for Molecular Medicine Berlin-Buch, 13125 Berlin, Germany, ${ }^{3}$ Vollum Institute, Oregon Health and Sciences University, Portland, \\ Oregon 97239, ${ }^{4}$ Laboratory of Genetics, The Salk Institute, La Jolla, California 92037, 5 Laboratory of Neuronal Plasticity, Leloir Institute, 1405 Buenos Aires, \\ Argentina, and ${ }^{\circ}$ Physiology Department, University of Freiburg, D-79104 Freiburg, Germany
}

Key words: neurogenesis; stem cell; development; plasticity; hippocampus; regeneration

New neurons are continuously added in the olfactory bulb and dentate gyrus of the hippocampus throughout adult life (Kempermann and Gage, 1999; Temple and Alvarez-Buylla, 1999; Schinder and Gage, 2004; Lledo and Saghatelyan, 2005; Ming and Song, 2005). This adult form of neurogenesis represents a previously unrecognized structural and functional plasticity in the mature mammalian brain, including in humans. Now it is well established that adult-born dentate granule cells (DGCs) can functionally integrate into the existing circuitry (Carlen et al., 2002; van Praag et al., 2002; Jessberger and Kempermann, 2003; Schmidt-Hieber et al., 2004). However, little is known about how that occurs and what is the contribution of new DGCs to the overall hippocampal function. Accumulating evidence suggests that adult neurogenesis is involved in many physiological and pathological conditions, such as learning and memory, epilepsy, mental disorders, and degenerative neurological diseases (Ming and Song, 2005). The impact of new neurons on the adult neuronal circuitry is determined by their physiological properties and synaptic connectivity. This mini-symposium presented at the 2005 Society for Neuroscience Meeting will provide insight into how newly generated neurons become synaptically integrated into the existing circuitry of the adult brain, with emphasis on the physiological properties of newborn DGCs in the hippocampus.

\section{Methodologies for the analysis of newborn neurons in the adult brain \\ Since the discovery of adult neurogenesis 40 years ago (Altman and Das, 1965), evidence that neurons generated in adulthood may be functional first came from studies of birds (Paton and Nottebohm, 1984) and only recently from mammals (van Praag et al., 2002; Belluzzi et al., 2003; Carleton et al., 2003). This is primarily attributable to technical difficulties in discriminating newly generated neurons from the large pool of mature neurons for physiological analysis. The traditional method uses incorpo-}

\footnotetext{
Received Aug. 16, 2005; revised Sept. 6, 2005; accepted Sept. 8, 2005.

We thank all colleagues and collaborators who have contributed to the works presented here.

Correspondence should be addressed to Hongjun Song, Institute for Cell Engineering, Department of Neurology, Johns Hopkins University School of Medicine, 733 North Broadway, BRB735, Baltimore, MD 21205. E-mail: shongju1@bs.jhmi.edu.

D0I:10.1523/JNEUROSCI.3452-05.2005

Copyright $\odot 2005$ Society for Neuroscience $\quad 0270-6474 / 05 / 2510366-03 \$ 15.00 / 0$
}

ration of nucleotide analogs [e.g., $\left[\mathrm{H}^{3}\right]$ thymidine and bromodeoxyuridine (BrdU)] into replicating DNA during the S-phase of the cell cycle (Miller and Nowakowski, 1988). The need of fixation makes it unsuitable for physiological analysis. Studies with BrdU labeling, however, provide evidence that a significant number of new DGCs are generated in adulthood, as much as $6 \%$ of the total size of the dentate granule cell layer (GCL) within 1 month in young adult rats (Cameron and McKay, 2001). Recently, several approaches have been developed to facilitate the identification of newly generated neurons in situ for physiological studies. The first approach uses retroviruses, based on the fact that the expression of transgenes from oncoretrovirus requires viral integration into the host genome, which only occurs during the cell cycle (Lewis and Emerman, 1994). When combined with a fluorescent marker such as green fluorescent protein (GFP), this approach provides adequate time resolution for birth dating and permanent marking of newborn neurons (van Praag et al., 2002). Retroviral vectors have now been developed to effectively express transgenes, short-hairpin RNAs, and site-specific recombinase for "single-cell genetic" analysis (Tashiro et al., 2004, 2005). The second approach uses transgenic mice harboring restricted expression of GFP in progenitors and/or immature neurons. In adult mice expressing GFP under the control of the regulatory regions of the nestin gene, labeled cells in the dentate gyrus include both neural progenitors (type 1 cells) and immature neurons (type 2a and 2b cells) (Yamaguchi et al., 2000; Filippov et al., 2003). In another line, GFP is expressed under the transcriptional control of proopiomelanocortin (POMC) genomic sequences (Overstreet et al., 2004b). Labeled cells do not express POMC mRNA or protein, but cryptic sequences in the transgene provide consistent expression in DGCs with immature characteristics, including high input resistance and expression of immature neuronal markers. BrdU colabeling indicates that the transient expression of GFP occurs when adult-generated cells are $\sim 2$ weeks postmitotic. The third approach is based on distinct membrane properties of newborn DGCs. Polysialylated neural cell adhesion molecule-positive (PSA-NCAM ${ }^{+}$) newborn DGCs exhibit very high input resistance (range from 1 to $20 \mathrm{G} \Omega$, depending on the age of the cell) compared with mature DGCs (Wang et al., 2000; Ambrogini et al., 2004; Schmidt-Hieber et al., 2004). Although each of these approaches has its advantages and caveats (Ming and Song, 2005), we are learning a great deal about 
the functional integration of new neurons in the adult brain using these techniques in combination with electrophysiology, $\mathrm{Ca}^{2+}$ imaging, and multiphoton time-lapse microscopy.

\section{Milestones of neuronal development in the adult hippocampus}

Adult neurogenesis represents a complete recapitulation of neuronal development in the mature brain, from fate specification of neural progenitors, migration and axon/dendritic targeting, to synaptogenesis of newborn neurons (Ming and Song, 2005). In the adult dentate gyrus, proliferating neural progenitors located in the subgranular zone give rise to new neurons, which then migrate a short distance into the inner GCL to become DGCs. Within 4-10 d, these new DGCs project their axons toward the CA3 region and contact pyramidal neurons and hilar interneurons (Hastings and Gould, 1999). Their dendrites extend toward the molecular layer and continue to elaborate for several weeks (van Praag et al., 2002). The first synaptic input, which is GABAergic, reaches newborn cells at the stage of nestin ${ }^{+}$nonradial precursor cells (type-2 cells) (Wang et al., 2005). These cells maintain their proliferative activity during the next stage and express doublecortin and PSA-NCAM (Kempermann et al., 2004). In adult POMC-enhanced GFP (EGFP) mice, labeled DGCs that express PSA-NCAM also receive GABAergic synaptic inputs (Overstreet Wadiche et al., 2005a). Glutamatergic synaptic transmission can be detected in some PSA-NCAM ${ }^{+}$DGCs after stimulation of the perforant pathway (Schmidt-Hieber et al., 2004). Dendritic spines, the major site of excitatory synaptic transmission, start to appear in new DGCs at 2-3 weeks after the cells are born in adults (Zhao et al., 2004). There is an initial peak of spine growth, which slows down and reaches a plateau at $\sim 8$ weeks. However, the density of mushroom spines continues to increase, indicating a prolonged structural modification of adultborn DGCs. Furthermore, the timing of functional maturation may be subject to modulation. After pilocarpine-induced seizures, EGFP-labeled DGCs in the adult POMC-EGFP mice exhibit elongated dendrites and functional glutamatergic synaptic inputs, suggesting that activity accelerates the functional integration of new neurons in the adult brain (Bromberg et al., 2004; Overstreet Wadiche et al., 2005b).

\section{Comparison of neuronal integration in the neonatal and adult brain}

During adult neurogenesis, new neurons have to integrate into the existing active circuitry formed by mature neurons. An interesting question arises as to whether the neuronal development process is different in the brain of neonatal, juvenile, and adult animals. Current evidence suggests that the morphological maturation and functional integration processes for new DGCs appear to be quite similar regardless of the age of the animal. Labeled DGCs of POMC-EGFP mice in neonates, juveniles, and adults exhibit similar physiological properties and sequence of synaptic integration, with GABAergic inputs being formed ahead of glutamatergic ones (Overstreet Wadiche et al., 2005a). GABAergic input to newborn DGCs has immature characteristics, including slow kinetics and depolarized reversal potentials (Overstreet Wadiche et al., 2005) (S. Ge and H. Song, unpublished results). Interestingly, GABAergic afferent connectivity with mature properties can only be observed after functional glutamatergic inputs have been established (S. Espósito, V. Piatti, and A. F. Schinder, unpublished results).

Because of significant differences in the environment between the neonatal and adult brains, we might expect to see some dif- ferences in the development and integration of new neurons. Indeed, analysis of POMC-EGFP mice show that the development of adult born DGCs is significantly delayed (Overstreet et al., 2004a). Accordingly, retroviral studies showed that spine formation starts much earlier in DGCs born during early postnatal development than those born in adults (C. Zhao and F. H. Gage, unpublished results).

\section{Unique properties associated with adult-born neurons in the dentate gyrus}

The adult hippocampal GCL constitutes a heterogeneous neuronal population that originates at three distinct developmental stages: late embryonic, early postnatal, and adult brain (Altman and Bayer, 1990). A critical question emerges: do all DGCs have the same function? That is, are they similar in terms of their intrinsic neuronal properties, afferent connectivity, and projections? Are there any special features for adult-born DGCs?

Recent studies showed that newborn DGCs exhibit distinct electrophysiological properties compared with their mature neighbors in the adult brain. Notably, newborn DGCs exhibit an enhanced excitability attributable to their high input resistance and the expression of low-threshold T-type $\mathrm{Ca}^{2+}$ channels (Ambrogini et al., 2004; Schmidt-Hieber et al., 2004). A small postsynaptic current $(\sim 10 \mathrm{pA})$ is sufficient for the cell to reach firing threshold (Schmidt-Hieber et al., 2004). The T-type $\mathrm{Ca}^{2+}$ channels in newborn DGCs are activated at a membrane potential of approximately $-56 \mathrm{mV}$ and can generate isolated lowthreshold $\mathrm{Ca}^{2+}$ spikes or boost overshooting action potentials (APs). Furthermore, they have a slow membrane time constant ( $\sim 120 \mathrm{~ms}$ ) and, therefore, a slow decay time course of the EPSPs, leading to effective temporal summation of excitatory synaptic inputs. The expression of the $\mathrm{Ca}^{2+}$-binding protein calbindin in the newborn neurons also appears to be much lower than in neighboring mature DGCs (Muller et al., 2005), indicating a low $\mathrm{Ca}^{2+}$ buffering capacity. Indeed, both $\mathrm{Ca}^{2+}$ spikes and APs evoke large dendritic $\mathrm{Ca}^{2+}$ transients. The decay time constant of these $\mathrm{Ca}^{2+}$ transients, measured with high- and low-affinity $\mathrm{Ca}^{2+}$ sensitive fluorescent dyes, is relatively slow, which might be attributable to low expression of $\mathrm{Ca}^{2+}$ pumps and $\mathrm{Na}^{+} / \mathrm{Ca}^{2+}$ exchangers (Stocca et al., 2005). This leads to effective temporal summation of $\mathrm{Ca}^{2+}$ signals, which might be important for the $\mathrm{Ca}^{2+}$-dependent regulation of fiber outgrowth and synaptic plasticity.

Newborn DGCs in adults indeed exhibit unique properties of synaptic plasticity (Schmidt-Hieber et al., 2004). During spatial exploration and other learning tasks, the entorhinal input fibers fire APs that are modulated by theta frequency $(4-8 \mathrm{~Hz})$. The induction of long-term potentiation (LTP) in DGCs was investigated by pairing short bursts of evoked EPSPs with small somatic current injections repeated at $5 \mathrm{~Hz}$. Interestingly, newly generated DGCs in adults need only to fire a single AP to effectively induce LTP, whereas mature DGCs need a short burst of 5-10 APs during each theta cycle (Schmidt-Hieber et al., 2004). Furthermore, paring EPSPs with subthreshold $\mathrm{Ca}^{2+}$ spikes leads to rapid long-term depression (LTD) of synaptic potentials in young neurons but not in mature DGCs (Bischofberger et al., 2004). Thus, newly generated DGCs appear to have a lower threshold for induction of LTP and LTD, leading to an enhanced bi-directional plasticity. In anesthetized animals in vivo, increased neurogenesis by voluntary exercise lowered the threshold for LTP of synaptic field potentials induced by theta burst stimulation (Farmer et al., 2004). Conversely, blockade of adult neurogenesis by $\gamma$-irradiation increased the threshold for LTP induc- 
tion (Snyder et al., 2001). Although voluntary exercise and $\gamma$-irradiation might have consequences in addition to modulation of neurogenesis, all of these data converge to support the conclusion that synaptic inputs to new DGCs have a lower threshold for synaptic plasticity. These findings have significant implications for the potential functions of adult neurogenesis. Future experiments will clarify whether the induction and expression mechanisms of enhanced plasticity are purely postsynaptic or whether the young neurons are able to turn on some more juvenile mechanisms inside the much older presynaptic fibers of mature neurons. Newly generated DGCs in the adult brain exhibit enhanced excitability and synaptic plasticity as long as they express PSA-NCAM (Schmidt-Hieber et al., 2004). Whether adult-born DGCs retain these properties and remain a distinct population beyond this time window needs to be determined.

\section{Conclusion}

Significant progress has been made in characterizing the physiological properties of new neurons and in understanding how they get synaptic inputs from mature neurons in the adult brain. In contrast, we know very little about the output of adult-born neurons. We also have to learn how environmental conditions influence their synaptic integration and how they, in turn, affect behavior. The molecular mechanisms underlying neuronal integration in the adult brain also remain to be identified. Although there is still significant work ahead, the present data indicate that adult neurogenesis is more than just an increase in the number of neurons. Rather, adult neurogenesis provides a continuous source of new neurons that appear to be qualitatively distinct computational units. Future studies of this striking form of adult plasticity will not only contribute to our understanding of the mechanisms and functional significance of neurogenesis in the adult mammalian brain but may also lead to novel strategies for cell-replacement therapy after injury and degenerative neurological diseases.

\section{References}

Altman J, Bayer SA (1990) Migration and distribution of two populations of hippocampal granule cell precursors during the perinatal and postnatal periods. J Comp Neurol 301:365-381.

Altman J, Das GD (1965) Post-natal origin of microneurones in the rat brain. Nature 207:953-956.

Ambrogini P, Lattanzi D, Ciuffoli S, Agostini D, Bertini L, Stocchi V, Santi S, Cuppini R (2004) Morpho-functional characterization of neuronal cells at different stages of maturation in granule cell layer of adult rat dentate gyrus. Brain Res 1017:21-31.

Belluzzi O, Benedusi M, Ackman J, LoTurco JJ (2003) Electrophysiological differentiation of new neurons in the olfactory bulb. J Neurosci 23:10411-10418.

Bischofberger J, Schmidt-Hieber C, Jonas P (2004) $\mathrm{Ca}^{2+}$ spikes and bidirectional synaptic plasticity in young hippocampal granule cells. Soc Neurosci Abstr 30:56.15.

Bromberg D, Overstreet LS, Westbrook GL (2004) Seizures accelerate functional integration of newly born granule neurons. Soc Neurosci Abstr 30:611.11.

Cameron HA, McKay RD (2001) Adult neurogenesis produces a large pool of new granule cells in the dentate gyrus. J Comp Neurol 435:406-417.

Carlen M, Cassidy RM, Brismar H, Smith GA, Enquist LW, Frisen J (2002) Functional integration of adult-born neurons. Curr Biol 12:606-608.

Carleton A, Petreanu LT, Lansford R, Alvarez-Buylla A, Lledo PM (2003) Becoming a new neuron in the adult olfactory bulb. Nat Neurosci 6:507-518.

Farmer J, Zhao X, van Praag H, Wodtke K, Gage FH, Christie BR (2004) Effects of voluntary exercise on synaptic plasticity and gene expression in the dentate gyrus of adult male Sprague-Dawley rats in vivo. Neuroscience 124:71-79.
Filippov V, Kronenberg G, Pivneva T, Reuter K, Steiner B, Wang LP, Yamaguchi M, Kettenmann H, Kempermann G (2003) Subpopulation of nestin-expressing progenitor cells in the adult murine hippocampus shows electrophysiological and morphological characteristics of astrocytes. Mol Cell Neurosci 23:373-382.

Hastings NB, Gould E (1999) Rapid extension of axons into the CA3 region by adult-generated granule cells. J Comp Neurol 413:146-154.

Jessberger S, Kempermann G (2003) Adult-born hippocampal neurons mature into activity-dependent responsiveness. Eur J Neurosci 18:2707-2712.

Kempermann G, Gage FH (1999) New nerve cells for the adult brain. Sci Am 280:48-53.

Kempermann G, Jessberger S, Steiner B, Kronenberg G (2004) Milestones of neuronal development in the adult hippocampus. Trends Neurosci 27:447-452.

Lewis PF, Emerman M (1994) Passage through mitosis is required for oncoretroviruses but not for the human immunodeficiency virus. J Virol 68:510-516.

Lledo PM, Saghatelyan A (2005) Integrating new neurons into the adult olfactory bulb: joining the network, life-death decisions, and the effects of sensory experience. Trends Neurosci 28:248-254.

Miller MW, Nowakowski RS (1988) Use of bromodeoxyuridineimmunohistochemistry to examine the proliferation, migration and time of origin of cells in the central nervous system. Brain Res 457:44-52.

Ming G-L, Song H (2005) Adult neurogenesis in the mammalian central nervous system. Annu Rev Neurosci 28:223-250.

Muller A, Kukley M, Stausberg P, Beck H, Muller W, Dietrich D (2005) Endogenous $\mathrm{Ca}^{2+}$ buffer concentration and $\mathrm{Ca}^{2+}$ microdomains in hippocampal neurons. J Neurosci 25:558-565.

Overstreet LS, Bensen AL, Westbrook GL (2004a) Delayed development of adult generated neurons. Soc Neurosci Abstr 30:155.6.

Overstreet LS, Hentges ST, Bumaschny VF, de Souza FS, Smart JL, Santangelo AM, Low MJ, Westbrook GL, Rubinstein M (2004b) A transgenic marker for newly born granule cells in dentate gyrus. J Neurosci 24:3251-3259.

Overstreet Wadiche LS, Bromberg DA, Bensen AL, Westbrook GL (2005a) GABAergic signaling to newborn neurons in dentate gyrus. J Neurophysiol, in press.

Overstreet Wadiche LS, Bensen AL, Bromberg DA, Westbrook GL (2005b) Network activity promotes maturation of adult-generated granule cells. Soc Neurosci Abstr 30:27.8.

Paton JA, Nottebohm FN (1984) Neurons generated in the adult brain are recruited into functional circuits. Science 225:1046-1048.

Schinder AF, Gage FH (2004) A hypothesis about the role of adult neurogenesis in hippocampal function. Physiology (Bethesda) 19:253-261.

Schmidt-Hieber C, Jonas P, Bischofberger J (2004) Enhanced synaptic plasticity in newly generated granule cells of the adult hippocampus. Nature 429:184-187.

Snyder JS, Kee N, Wojtowicz JM (2001) Effects of adult neurogenesis on synaptic plasticity in the rat dentate gyrus. J Neurophysiol 85:2423-2431.

Stocca G, Jonas P, Bischofberger J (2005) Dendritic calcium signaling n young hippocampal granule cells. Soc Neurosci Abstr 31:738.11.

Tashiro A, Zhao C, Gage FH (2004) Retrovirus-mediated gene knockout technique in adult newborn neurons. Soc Neurosci Abstr 30:607.17.

Tashiro A, Zhao C, Gage FH (2005) Competition for survival: NMDA receptor-mediated selective integration of adult new neurons in the dentate gyrus. Soc Neurosci Abstr 31:141.1.

Temple S, Alvarez-Buylla A (1999) Stem cells in the adult mammalian central nervous system. Curr Opin Neurobiol 9:135-141.

van Praag H, Schinder AF, Christie BR, Toni N, Palmer TD, Gage FH (2002) Functional neurogenesis in the adult hippocampus. Nature 415:1030-1034.

Wang LP, Kempermann G, Kettenmann H (2005) A subpopulation of precursor cells in the mouse dentate gyrus receives synaptic GABAergic input. Mol Cell Neurosci 29:181-189.

Wang S, Scott BW, Wojtowicz JM (2000) Heterogenous properties of dentate granule neurons in the adult rat. J Neurobiol 42:248-257.

Yamaguchi M, Saito H, Suzuki M, Mori K (2000) Visualization of neurogenesis in the central nervous system using nestin promoter-GFP transgenic mice. NeuroReport 11:1991-1996.

Zhao C, Tashiro A, Ming G-l, Gage FH (2004) The maturation of newborn granule neurons in the adult mouse hippocampus. Soc Neurosci Abstr 30:607.18. 